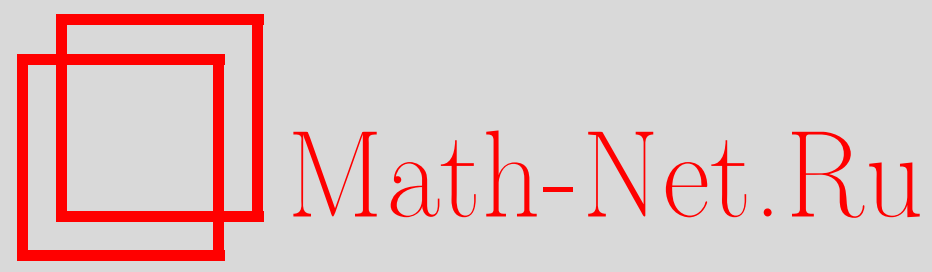

В. П. Деревенский, Интегрируемые системы линейных обыкновенных дифференциальных уравнений общего порядка, Матем. заметки, 2004, том 76, выпуск 1, 52-65

DOI: https://doi.org/10.4213/mzm86

Использование Общероссийского математического портала Math-Net.Ru подразумевает, что вы прочитали и согласны с пользовательским соглашением http://www.mathnet.ru/rus/agreement

Параметры загрузки:

IP : 54.166 .219 .16

26 апреля 2023 г., 16:51:50 


\title{
ИНТЕГРИРУЕМЫЕ СИСТЕМЫ ЛИНЕЙНЫХ ОБЫКНОВЕННЫХ ДИФФЕРЕНЦИАЛЬНЫХ УРАВНЕНИЙ ОБЩЕГО ПОРЯДКА
}

\section{В. П. Деревенский}

\begin{abstract}
В работе определяются достаточные условия разрешимости в квадратурах систем линейных обыкновенных дифференциальных уравнений высших порядков с переменньми коэффициентами. Обобщаются на случай таких систем методы вариации постоянных уравнений высших порядков и систем уравнений первого порядка. Результаты получены путем сведения рассматриваемых систем к матричным уравнениям высших порядков, исследуемых методами теории алгебр Ли.
\end{abstract}

Библиограффия: 8 названий.

В работе определяются достаточные условия разрешимости в квадратурах систем линейных обыкновенных дифференциальных уравнений (СЛОДУ) высших порядков с переменными коэффициентами.

1. Постановка задачи. Пусть $M_{n}$ - множество $(n \times n)$-матриц над действительным полем $R, M_{n}^{k}(t)$ - совокупность элементов $M_{n}$, компоненты которых являются функциями $t$ класса $C^{k}(t), \mathbf{E}$ - единица $M_{n}, L_{N}$-представление в $M_{n} N$-мерной алгебры Ли с базисом $\left(\mathbf{E}_{\alpha}\right)$ и структурными константами $C_{\alpha \beta}^{\gamma}\left(\alpha, \beta, \gamma=1, \ldots, N \leqslant n^{2}<\infty\right)$. Предположим, что $R_{K}$ - радикал $L_{N}(K \leqslant N)$, т.е. наибольшая разрешимая подалгебра ее, обладающая (по определению) свойствами:

1) $\left[R_{K} L_{N}\right] \subset R_{K}$,

2) $\exists M<\infty: R_{K}^{(M)}=0, R_{K}^{(m)} \equiv\left[R_{K}^{(m-1)} R_{K}^{(m-1)}\right]$,

где [AB]- коммутант множеств $A$ и $B$. Договоримся нумеровать базисные матрицы $L_{N}$ так, чтобы $\mathbf{E}_{\alpha} \in R_{K}$ при $\alpha=1, \ldots, K, \mathbf{E}_{\alpha} \in U_{N-K} \equiv L_{N} \backslash R_{K}$ при $\alpha=K+1, \ldots, N$.

Рассмотрим нормальную систему ЛОДУ $K$-го порядка (СЛОДУ.К)

$$
\sum_{s=K}^{0} A_{i s}^{j} x_{j}^{(s)}=f_{i}, \quad A_{i K}^{j}=\delta_{i}^{j}, \quad i, j=1, \ldots, n,
$$

где матрицы $\mathbf{A}_{s} \equiv\left(A_{i s}^{j}\right) \in M_{n}^{0}(t),\left(\delta_{i}^{j}\right)=\mathbf{E}$; по повторяющимся верхним и нижним индексам производится суммирование, $t \in T$, а $T$ - область существования $A_{i s}^{j}$. Метод сведения скалярных ЛОДУ.К к линейной системе первого порядка $[1$, c. 139] позволяет сформулировать 
ПРЕДЛОЖЕНИЕ 1. Система (1) әквивалентна системе Кп ЛОДУ.1

$$
\dot{y}_{p}=B_{p}^{q} y_{q}+C_{p}, \quad p, q=1, \ldots, K n,
$$

с матричным коэффициентом $\mathscr{B} \equiv\left(B_{p}^{q}\right) \in M_{K n}^{0}(t)$, состоящим из $n^{2}(K \times K)$-мерных блоков $\mathscr{B}_{i}^{j}$ вида

$$
\begin{gathered}
\mathscr{B}_{i}^{i}=\left(\begin{array}{ccccc}
0 & 1 & 0 & \ldots & 0 \\
0 & 0 & 1 & \ldots & 0 \\
\ldots \ldots & \ldots & \ldots & \ldots & \ldots \\
0 & 0 & 0 & \ldots & 1 \\
-A_{i 0}^{i} & -A_{i 1}^{i} & -A_{i 2}^{i} & \ldots & -A_{i K-1}^{i}
\end{array}\right), \\
\mathscr{B}_{i}^{j}=\left(\begin{array}{cccc}
0 & 0 & \ldots & 0 \\
0 & 0 & \ldots & 0 \\
\ldots \ldots & \ldots & \ldots \\
0 & 0 & \ldots & 0 \\
-A_{i 0}^{j} & -A_{i 1}^{j} & \ldots & -A_{i K-1}^{j}
\end{array}\right)
\end{gathered}
$$

$a C_{p}=0, p \neq K i, C_{K i}=f_{i}$.

ДокАЗАТЕльство. Введем новые неизвестные

$$
y_{p} \equiv y_{K(i-1)+k} \equiv x_{i}^{(k-i)}, \quad k=1, \ldots, K .
$$

Тогда для $k \neq K y_{p}=\dot{y}_{p-1}$, а для $k=K$, т.е. $p=K i$, система (1) позволяет определить $\dot{y}_{p}$ в виде

$$
\dot{y}_{p}=f_{p}-\sum_{s=0}^{K-1}\left(A_{p s}^{1} y_{s}+A_{p s}^{2} y_{K+s}+\cdots+A_{p s}^{n} y_{(n-1) K-s}\right) .
$$

Совокупность этих систем и определяет в $M_{K n}^{0}$ СЛОДУ.1 (2).

Из предложения 1 вытекает

СлЕдСТВИЕ. В области Т существует решение системы (1), которое при задании начальных условий является единственным.

Действительно, так как система (1) эквивалентна системе (2), для которой в $T$ всегда сушествует единственное решение [1, с. 22], оно является решением и системы (1).

В определении системы (1) существенно условие $\mathbf{A}_{K}=\mathbf{E}$. Оно не только нормализует каждое из уравнений С ЛОДУ.К, но и обеспечивает одновременную разрешимость их относительно производных высших порядков искомых функций. Это предполагает рассмотрение нормальных систем общего порядка. В противном случае вопросы существования и единственности решений системы (1) остаются открытыми.

При положительном ответе на эти вопросы возникает главная проблема теории дифференциальных уравнений: проблема интегрирования СЛОДУ.К в квадратурах. При этом известно,что даже отдельные ЛОДУ.2 не интегрируются в них в общем случае. Для них даются лишш достаточные условия разрешимости и соответствуюшие им методы интегрирования. Естественно, и для СЛОДУ.К возникает проблема определения условий и методов разрешимости их. Так что в настояшей работе ставится задача об определении достаточных условий интегрируемости в квадратурах нормальных СЛОДУ.К и соответствующих методов нахождения их решений. 
2. Сведение решения неоднородньх систем к решению однородных в случае систем (1) является, очевидно, задачей №1. Ее решение должно обобщить классические "методы вариации постоянньх" (МВП) неоднородных СЛОДУ.1 и уравнений высших порядков $[1$, с. 135,143$]$ на случай СЛОДУ.К.

Простейший вариант такого обобщения, очевидно, следует из предложения 1.

ПРЕДЛОЖЕНИЕ 2. Если $\mathbf{Y} \equiv\left(Y_{p}^{q}\right)$ - фундаментальная система решений однородной системы (2),

$$
\dot{Y}_{p}^{q}=B_{p}^{r} Y_{r}^{q}, \quad p, q, r=1, \ldots, K n
$$

то общим решением системы (1) является

$$
x_{i}=Y_{K(i-1)+1}^{q} \int\left(\mathbf{Y}^{-1}\right) q^{K j} f_{j} d t, \quad i, j=1, \ldots, n .
$$

ДокАЗАТЕЛЬСтво. Из (4) следует, что при $k=1 x_{i}=y_{K(i-1)+1}$. Следовательно, общее решение системы (2) определяется в виде

$$
y_{p}=Y_{p}^{q} \int\left(\mathbf{Y}^{-1}\right)_{q}^{r} C_{r} d t
$$

Таким образом, $x_{i}=y_{K(i-1)+1}=Y_{K(i-1)+1}^{q} \int\left(\mathbf{Y}^{-1}\right)_{q}^{r} C_{r} d t$. Однако, ненулевыми компонентами $K n$-мерного вектора $\left(C_{r}\right)$ являются $C_{K i}=f_{i}$, что и позволяет из последнего равенства получить (6). Неопределенньй интеграл правой части этой формулы задается с точностью до постоянного $K n$-мерного вектора. Так что эквивалентность систем (1) и (2) и обшность решения (7) второй из них обеспечивает общность решения (6) СЛОДУ.К.

Приведенное утверждение имеет несколько недостатков, важнейшими из которых являются резко возрастающая трудоемкость работы в $M_{K n}$ сравнительно с $M_{n}$ и потеря наглядной зависимости решения системы (1) от ее матричных параметров. Поэтому целесообразно дать для нее аналоги известных МВП. Так обобщение использованного выше метода для систем ЛОДУ.1 дает

ТЕОрема 1. Общим решением системы (1) является

$$
x_{i}=x_{K i}^{j} \int x_{K-1 j}^{l} \int x_{K-r l}^{m} \cdots \int x_{1 m}^{p} \int\left(\prod_{k=K}^{1} \mathbf{X}_{k}^{-1}\right)_{p}^{q} f_{q} d t \cdots d t
$$

әде $i, j, l, m, p, q=1, \ldots, n, \mathbf{X}_{k} \equiv\left(x_{k i}^{j}\right) \in M_{n}^{k}(t)$ - невырожденные решения матричных левосторонних уравнений

$$
\sum_{s=K-q}^{0} \mathbf{A}_{q, s} \mathbf{X}_{K-q}^{(s)}=0, \quad q=0, \ldots, K-1, \quad\left|\mathbf{X}_{k}\right| \neq 0
$$

параметры которых определяются рекуррентными соотношениями

$$
\mathbf{A}_{q, s}=\sum_{i=0}^{K-q-s} C_{K-q-i+1}^{s+1} \mathbf{A}_{q-1, K-q-i+1} \mathbf{X}_{K-q+1}^{(K-q-s-i)}, \quad C_{m}^{n}=\frac{m !}{n !(m-n) !} .
$$


ДокАЗАТЕЛЬСтво. Запишем матричное левостороннее ЛОДУ.К (МЛЛОДУ.К),которому удовлетворяют любые $n$ решений системы (1), перенумерованные верхним индексом $j, \mathbf{X} \equiv\left(x_{i}^{j}\right)$ :

$$
\sum_{s=K}^{0} \mathbf{A}_{s} \mathbf{X}^{(s)}=F, \quad \mathbf{A}_{K}=\mathbf{E},
$$

где матрица $\mathbf{F} \equiv\left(f_{i}^{j}\right)$ имеет одинаковые элементы строк, $f_{i}^{j}=f_{i}$. Пусть

$$
\mathbf{X}=\mathbf{X}_{K} \int \mathbf{Y}_{1} d t, \quad \mathbf{X}_{K} \in M_{n}^{K}(t), \quad \mathbf{Y}_{1} \in M_{n}^{K-1}(t)
$$

где $\mathbf{X}_{K}$ - невырожденное решение однородного уравнения (11),

$$
\sum_{s=K}^{0} \mathbf{A}_{s} \mathbf{X}_{K}^{(s)}=0, \quad\left|\mathbf{X}_{K}\right| \neq 0 .
$$

Найдем ограничение, накладываемое на $\mathbf{Y}_{1}$ этим предположением. Для этого методом индукции убедимся в справедливости формулы Лейбница кратного дифференцирования матричного произведения.

Очевидно, для $k=1,2$

$$
(\mathbf{X Y})^{(k)}=\sum_{s=0}^{k} C_{k}^{s} \mathbf{X}^{(s)} \mathbf{Y}^{(k-s)}
$$

Допустим, что это соотношение справедливо и для $k>2$. Тогда

$$
\begin{aligned}
(\mathbf{X Y})^{(k+1)} & =\frac{d}{d t}(\mathbf{X Y})^{k}=\sum_{s=0}^{k} C_{k}^{s}\left(\mathbf{X}^{(s+1)} \mathbf{Y}^{(k-s)}+\mathbf{X}^{(s)} \mathbf{Y}^{(k-s+1)}\right) \\
& =\mathbf{X}^{(k+1)} \mathbf{Y}+\sum_{s=0}^{k}\left(C_{k}^{s}+C_{k}^{s+1}\right) \mathbf{X}^{(s+1)} \mathbf{Y}^{(k-s)}+\mathbf{X} \mathbf{Y}^{(k+1)}
\end{aligned}
$$

Однако [2, c. 11], $C_{k}^{s}+C_{k}^{s+1}=C_{k+1}^{s+1}$. Следовательно, формула Лейбница справедлива и для матричного произведения.

Таким образом, подставляя $X$ вида (12) в (11), получаем

$$
\sum_{s=K}^{0} \mathbf{A}_{s}\left[\sum_{r=0}^{s} C_{s}^{r} \mathbf{X}_{K}^{(r)}\left(\int \mathbf{Y}_{1} d t\right)^{(s-r)}\right]=\mathbf{F} .
$$

В силу (13) сумма коэффициентов при $\int \mathbf{Y}_{1} d t$ в уравнении (14) равна нулю. Это делает его линейным уравнением $(K-1)$-го порядка относительно $\mathbf{Y}_{1}$. Складывая коэффициенты при производных общего порядка матрицы $\mathbf{Y}_{1}$, имеем

$$
\sum_{s=K-1}^{0}\left(\sum_{r=0}^{s} C_{K-r}^{K-s} \mathbf{A}_{K-r} \mathbf{X}_{K}^{(s-r)}\right) \mathbf{Y}_{1}^{(K-s-1)}=\mathbf{F} .
$$


Если ввести обозначения

$$
\mathbf{A}_{1, s} \equiv \sum_{r=0}^{s} C_{K-r}^{K-s} \mathbf{A}_{0, K-r} \mathbf{X}_{K}^{(s-r)}, \quad \mathbf{A}_{0, s} \equiv \mathbf{A}_{s}, \quad \mathbf{Y}_{0} \equiv \mathbf{X}
$$

то исходное матричное уравнение (11) записьвается как

$$
\sum_{s=K}^{0} \mathbf{A}_{0, s} \mathbf{Y}_{0}^{(s)}=\mathbf{F}
$$

а его решение - в виде

$$
\mathbf{Y}_{0}=\mathbf{X}_{K} \int \mathbf{Y}_{1} d t, \quad \sum_{s=K}^{0} \mathbf{A}_{0, s} \mathbf{X}_{K}^{(s)}=0, \quad\left|\mathbf{X}_{K}\right| \neq 0
$$

где $\mathbf{Y}_{1}$ удовлетворяют неоднородному МЛЛОДУ.К-1

$$
\sum_{s=K-1}^{0} \mathbf{A}_{1, s} \mathbf{Y}_{1}^{(s)}=\mathbf{F}
$$

Коэффициенты этого уравнения зависят от параметров исходного матричного уравнения и решения однородного МЛЛОДУ.К (18). Эта зависимость дается формулами (16). Так как (17) и (19) отличаются лиш величиною $K$ и обозначениями матричных коэф фициентов, процедуру понижения порядка неоднородного МЛЛОДУ можно повторить. Обозначим, аналогично (18),

$$
\begin{gathered}
\mathbf{Y}_{1} \equiv \mathbf{X}_{K-1} \int \mathbf{Y}_{2} d t, \sum_{s=K-1}^{(s)} \mathbf{A}_{1, s} \mathbf{X}_{K-1}^{(s)}=0 \\
\left|\mathbf{X}_{K-1}\right| \neq 0, \quad \sum_{s=K-2}^{0} \mathbf{A}_{2, s} \mathbf{Y}_{2}^{(s)}=\mathbf{F}
\end{gathered}
$$

где $\mathbf{A}_{2, s}$ определяются через $\mathbf{A}_{1, s}$ соотношениями (16). Равенства (20) получаются из формул (18) и (19) заменой $\left(\mathbf{X}_{K}, \mathbf{Y}_{0}, \mathbf{Y}_{1}\right) \rightarrow\left(\mathbf{X}_{K-1}, \mathbf{Y}_{1}, \mathbf{Y}_{2}\right)$. При этом, в отличие от уравнения (11), которое имеет единичный коэффициент при старшей производной искомой матрицы, МЛЛОДУ.К $-i(i=1,2)$ не являются нормальньми; коэффициентами при $\mathbf{Y}_{i}^{(K-i)}$ являются $\mathbf{X}_{K}$ и $\mathbf{X}_{K-1}$, соответственно.

Таким образом, в процессе редуцирования порядков неоднородных матричных ЛОДУ решение уравнения (11) сводится к решению $K$ однородных МЛЛОДУ. $k, k=$ $1, \ldots, K$ вида (9). Коэффициенты этих уравнений определяются через все коэффициенты и решения однородных уравнений более высоких порядков. Их связывают рекуррентные формулы (10). Последним в этой череде уравнений будет алгебраическое матричное уравнение $\mathbf{X}_{1} \mathbf{X}_{2} \cdots \mathbf{X}_{K} \mathbf{Y}_{K}=\mathbf{F}$. В силу невырожденности $\mathbf{X}_{k}$ вьполнено $\mathbf{Y}_{K}=\left(\prod_{k=1}^{K} \mathbf{X}_{k}\right)^{-1} \mathbf{F}$. Следовательно,

$$
\mathbf{X}=\mathbf{X}_{K} \int \mathbf{X}_{K-1} \cdots \int \mathbf{X}_{1} \int \mathbf{X}_{K}^{-1} \mathbf{X}_{K-1}^{-1} \cdots \mathbf{X}_{1}^{-1} \mathbf{F} d t \cdots d t
$$


что позволяет записать решение системы (1) в виде любого столбца этой матрицы. Отбрасьвая в выбранном столбце его номер, можно представить расписанноепо компонентам равенство $(21)$ в виде $(8)$.

Покажем, что решение (8) является общим. Для этого достаточно убедиться в том, что $\mathbf{X}$ вида (21) является общим решением уравнения (11).

Задавая начальные данные уравнения (11) в виде

$$
\mathbf{X}^{(q)}\left(t_{0}\right)=\mathbf{R}_{q}, \quad \dot{\mathbf{R}}_{q}=0, \quad q=0, \ldots, K-1,
$$

решение (21) можно представить как

$$
\begin{aligned}
\mathbf{X}= & \mathbf{X}_{K}\left(\int _ { t _ { 0 } } ^ { t } \mathbf { X } _ { K - 1 } \left(\int _ { t _ { 0 } } ^ { t _ { 1 } } \mathbf { X } _ { K - 2 } \cdots \left(\int _ { t _ { 0 } } ^ { t _ { K - 2 } } \mathbf { X } _ { 1 } \left(\int_{t_{0}}^{t_{K-1}} \mathbf{X}_{K}^{-1} \mathbf{X}_{K-1}^{-1} \cdots \mathbf{X}_{1}^{-1} \mathbf{F} d t_{K}\right.\right.\right.\right. \\
& \left.\left.\left.\left.\left.+\mathbf{Q}_{K}\right) d t_{K-1}+\mathbf{Q}_{K-1}\right) \cdots\right) d t_{2}+\mathbf{Q}_{2}\right) d t_{1}+\mathbf{Q}_{1}\right), \quad \dot{\mathbf{Q}}_{k}=0 .
\end{aligned}
$$

Следовательно,

$$
\left(\mathbf{X}\left(t_{0}\right)=\mathbf{R}_{0}\right) \Longrightarrow\left(\mathbf{X}_{K}\left(t_{0}\right) \mathbf{Q}_{1}=\mathbf{R}_{0}\right) \Longrightarrow\left(\mathbf{Q}_{1}=\mathbf{X}_{K}^{-1}\left(t_{0}\right) \mathbf{R}_{0}\right) .
$$

Беря производную от $\mathbf{X}$, мы можем наложить условие на $\mathbf{Q}_{2}$ :

$$
\begin{aligned}
\left(\dot{\mathbf{X}}\left(t_{0}\right)=\mathbf{R}_{1}\right) & \Longrightarrow\left(\mathbf{X}_{K}\left(t_{0}\right) \mathbf{Q}_{1}+\mathbf{X}_{K}\left(t_{0}\right) \mathbf{X}_{K-1}\left(t_{0}\right) \mathbf{Q}_{2}=\mathbf{R}_{1}\right) \\
& \Longrightarrow\left(\mathbf{Q}_{2}=\mathbf{X}_{K-1}^{-1}\left(t_{0}\right) \mathbf{X}_{K}^{-1}\left(t_{0}\right)\left(\mathbf{R}_{1}-\dot{\mathbf{X}}_{K}\left(t_{0}\right) \mathbf{Q}_{1}\right)\right) .
\end{aligned}
$$

Продолжив этот процесс, мы получаем алгебраическую линейную треугольную матричную систему, из которой легко находятся $\mathbf{Q}_{k}$.

Таким образом, классический МВП МЛЛОДУ.1 обобщается на случай систем уравнений высших порядков формулой (8). Сформулируем иллюстративное

СлЕДСТВИЕ. Общим решением систем

$$
\ddot{x}_{i}+A_{i 1}^{j} \dot{x}_{j}+A_{i 0}^{j} x_{j}=f_{i}, \quad i, j=1, \ldots, n, \quad \mathbf{A}_{s} \equiv\left(A_{i s}^{j}\right) \in M_{n}^{0}(t), \quad s=0, \ldots, 2,
$$

$u$

$$
\dddot{x}_{i}+A_{i 2}^{j} \ddot{x}_{j}+A_{i 1}^{j} \dot{x}_{j}+A_{i 0}^{j} x_{j}=f_{i}
$$

являются, соответственно,

$$
\begin{gathered}
x_{i}=x_{2 i}^{j} \int x_{1 j}^{l} \int\left(\mathbf{X}_{2}^{-1} \mathbf{X}_{1}^{-1}\right) l^{m} f_{m} d t d t, \quad \mathbf{X}_{k} \equiv\left(x_{k i}^{j}\right) \\
\ddot{\mathbf{X}}_{2}+\mathbf{A}_{1} \dot{\mathbf{X}}_{2}+\mathbf{A}_{0} \mathbf{X}_{2}=0, \quad \mathbf{X}_{2} \dot{\mathbf{X}}_{1}+\left(2 \dot{\mathbf{X}}_{2}+\mathbf{A}_{1} \mathbf{X}_{2}\right) \mathbf{X}_{1}=0, \quad\left|\mathbf{X}_{k}\right| \neq 0
\end{gathered}
$$

$u$

$$
\begin{gathered}
x_{i}=x_{3 i}^{j} \int x_{2 j}^{l} \int x_{1 l}^{m} \int\left(\mathbf{X}_{3}^{-1} \mathbf{X}_{2}^{-1} \mathbf{X}_{1}^{-1}\right) m^{p} f_{p} d t d t d t \\
\dddot{\mathbf{X}}_{3}+\mathbf{A}_{2} \ddot{\mathbf{X}}_{3}+\mathbf{A}_{1} \dot{\mathbf{X}}_{3}+\mathbf{A}_{0} \mathbf{X}_{3}=0 \\
\mathbf{X}_{3} \ddot{\mathbf{X}}_{2}+\left(3 \dot{\mathbf{X}}_{3}+\mathbf{A}_{2} \mathbf{X}_{3}\right) \dot{\mathbf{X}}_{2}+\left(3 \ddot{\mathbf{X}}_{3}+2 \mathbf{A}_{2} \dot{\mathbf{X}}_{3}+\mathbf{A}_{1} \mathbf{X}_{3}\right) \mathbf{X}_{2}=0 \\
\mathbf{X}_{2} \mathbf{X}_{3} \dot{\mathbf{X}}_{1}+\left(2 \mathbf{X}_{3} \dot{\mathbf{X}}_{2}+3 \dot{\mathbf{X}}_{3} \mathbf{X}_{2}+\mathbf{A}_{2} \mathbf{X}_{3} \mathbf{X}_{2}\right) \mathbf{X}_{1}=0
\end{gathered}
$$


ДоказАтЕЛЬСтво. Если в теореме $1 K=2$, то $q=0,1$. Для $q=0 s=2,1,0$, $\mathbf{A}_{0, s}=\mathbf{A}_{s}$, что и определяет МЛЛОДУ.2 в (25). Для $q=1 s=1,0$, а $\mathbf{A}_{1, s}$ определяются формулой (10): $\mathbf{A}_{1,1}=C_{2}^{2} \mathbf{A}_{0,2} \mathbf{X}_{2}=\mathbf{X}_{2}, \mathbf{A}_{1,0}=\sum_{i=0}^{1} C_{2-i}^{1} \mathbf{A}_{0,2-i} \mathbf{X}_{2}^{(1-i)}=2 \dot{\mathbf{X}}_{2}+$ $\mathbf{A}_{1} \mathbf{X}_{2}$. Если $K=3$, то $q=0,1,2 ;(q=0) \Longrightarrow(s=3, \ldots, 0)$, что определяет в (26) однородное уравнение 3 -го порядка. Для $q=1 s=2, \ldots, 0$, и согласно (10) мы имеем

$$
\begin{aligned}
& \mathbf{A}_{1,2}=C_{3}^{3} \mathbf{A}_{0,3} \mathbf{X}_{3}=\mathbf{X}_{3}, \quad \mathbf{A}_{1,1}=\sum_{i=0}^{1} C_{3-i}^{2} \mathbf{X}_{3}^{(1-i)}=3 \dot{\mathbf{X}}_{3}+\mathbf{A}_{2} \mathbf{X}_{3}, \\
& \mathbf{A}_{1,0}=\sum_{i=0}^{2} C_{3-i}^{1} \mathbf{A}_{0,3-i} \mathbf{X}_{3}^{(2-i)}=3 \ddot{\mathbf{X}}_{3}+2 \mathbf{A}_{2} \dot{\mathbf{X}}_{3} \mathbf{A}_{1} \mathbf{X}_{3} .
\end{aligned}
$$

Эти коэффициенты и стоят при $X_{2}^{(s)}$ во втором матричном уравнении (26). Для $q=2$ $s=1,0$, так что согласно (10)

$$
\begin{aligned}
& \mathbf{A}_{2,1}=C_{2}^{2} \mathbf{A}_{1,2} \mathbf{X}_{2}=\mathbf{X}_{3} \mathbf{X}_{2}, \\
& \mathbf{A}_{2,0}=\sum_{i=0}^{1} C_{2-i}^{1} \mathbf{A}_{1,2-i} \mathbf{X}_{2}^{(1-i)}=2 \mathbf{X}_{3} \dot{\mathbf{X}}_{2}+\left(3 \dot{\mathbf{X}}_{3}+\mathbf{A}_{2} \mathbf{X}_{3}\right) \mathbf{X}_{2} .
\end{aligned}
$$

Это и определяет МЛЛОДУ.1 в (26).

Второй вариант метода вариации постоянных для (1) возникает как обобщение на матричный случай МВП скалярных ЛОДУ высших порядков. Для реализации его необходимо использовать операторы Гаусса, позволяюшие решать линейные алгебраические матричные системы [3]. Под левым оператором Гаусса подразумевается линейньй оператор $\Gamma\left(\mathbf{A}_{i}\right)$, определенный на последовательности невырожденных матриц $\left(\mathbf{A}_{i}\right)$, $i=1, \ldots, I$, который действует на другую матричную последовательность $\left(\mathbf{Y}_{j}\right), j=$ $1, \ldots, J$, и перемножается по правилам

$$
\begin{aligned}
\Gamma\left(\mathbf{A}_{i}\right) \mathbf{Y}_{j} & \equiv \mathbf{Y}_{j}-\mathbf{A}_{j}\left(\mathbf{A}_{i}\right)^{-1} \mathbf{Y}_{i} \\
\Gamma\left(\mathbf{B}_{k}\right) \Gamma\left(\mathbf{A}_{i}\right) \mathbf{Y}_{j} & \equiv \Gamma\left(\Gamma\left(\mathbf{A}_{i}\right) \mathbf{B}_{k}\right)\left(\Gamma\left(\mathbf{A}_{i}\right) \mathbf{Y}_{j}\right) .
\end{aligned}
$$

В символах этих операторов решение системы матричных левосторонних линейных уравнений (СМАЛЛУ)

$$
\mathbf{A}_{i}^{j} \mathbf{X}_{j}=\mathbf{B}_{i}: \quad i, j=1, \ldots, K,
$$

где, как и вьше, по повторяюшимся верхним и нижним индексам производится суммирование, дается формулой

$$
\begin{gathered}
\mathbf{X}_{i}=\left[\prod_{k=1}^{i-1} \Gamma\left(\mathbf{A}_{k}^{k}\right) \mathbf{A}_{i}^{i}\right]^{-1} \prod_{\tau=K}^{i+1} \Gamma\left(\mathbf{A}_{\tau}^{\tau}\right)\left[\prod_{k=1}^{i-1} \Gamma\left(\mathbf{A}_{k}^{k}\right) \mathbf{B}_{i}\right] \equiv W_{i}(\mathbf{A}, \mathbf{B}), \\
\mathbf{A} \equiv\left(A_{i}^{j}\right), \quad \mathbf{B} \equiv\left(B_{i}\right) .
\end{gathered}
$$

Это соотношение предполагает невырожденность СМАЛЛУ, что определяется условиem

$$
d(\mathbf{A}) \equiv|\mathscr{D}(\mathbf{A})| \equiv\left|\mathbf{A}_{1}^{1} \prod_{i=2}^{K-1}\left(\prod_{k=i-1}^{1} \Gamma\left(\mathbf{A}_{i-k}^{i-k}\right) \mathbf{A}_{i}^{i}\right)\right| \neq 0 .
$$

С помошью операторов Гаусса доказывается следуюшая 
Теорема 2. Общим решением системы (1) является colon $\mathbf{X}$, где

$$
\begin{gathered}
\mathbf{X}=\sum_{k=1}^{K} \mathbf{X}_{k} \mathbf{C}_{k}, \quad \sum_{s=K}^{0} \mathbf{A}_{s} \mathbf{X}_{k}^{(s)}=0 \\
\mathbf{C}_{1}=-\int \mathbf{X}_{1}^{-1} \sum_{i=2}^{K} \mathbf{X}_{i} \mathbf{C}_{i} d t, \quad \mathbf{C}_{K}=\int\left[\prod_{m=1}^{K-1} \Gamma\left(\mathbf{X}_{m}^{(m-1)}\right) \mathbf{X}_{K}^{(K-1)}\right]^{-1} \mathbf{F} d t, \\
\mathbf{C}_{l}=-\int\left[\prod_{m=1}^{l-1} \Gamma\left(\mathbf{X}_{m}^{(m-1)}\right) \mathbf{X}_{l}^{(l-1)}\right]^{-1} \sum_{i=l+1}^{K}\left[\prod_{m=1}^{l-1} \Gamma\left(\mathbf{X}_{m}^{(m-1)}\right) \mathbf{X}_{i}^{(l-1)}\right] \dot{\mathbf{C}}_{i} d t
\end{gathered}
$$

анумерачия $\mathbf{X}_{k}$ такова, что

$$
d(\mathbf{V}) \neq 0, \quad \mathbf{V} \equiv\left(\mathbf{X}_{k}^{(q)}\right), \quad k=1, \ldots, K, \quad q=0, \ldots, K-1 .
$$

ДокАЗАТЕЛЬСтво. Так как решения системы (1) и матричного уравнения (11) связаны соотношением $\left(x_{i}\right)=$ colon $\mathbf{X}$, достаточно показать, что $\mathbf{X}$ вида (31) является общим решением МЛЛОДУ. Для этого рассмотрим фундаментальную систему решений однородного уравнения $(11)\left(\mathbf{X}_{k}\right)$, т.е. совокупность $K$ решений с такой нумерацией их, при которой вьполняется условие (33). Предположим, что $\mathbf{X}$ вида (31) удовлетворяет условиям

$$
\mathbf{X}^{(q)}=\sum_{k=1}^{K} \mathbf{X}_{k}^{(q)} \mathbf{C}_{k}, \quad q=1, \ldots, K-1 .
$$

Последовательно дифференцируя $\mathbf{X} K-2$ раз, легко убедиться в том, что совокупность этих требований накладьвает на $\mathbf{C}_{k}$ ряд ограничений:

$$
\sum_{k=1}^{K} \mathbf{X}_{k}^{(q)} \dot{\mathbf{C}}_{k}=0, \quad q=0, \ldots, K-2 .
$$

Подставим $\mathbf{X}_{k}$ в (11). Тогда

$$
\begin{aligned}
\sum_{s=K}^{0} \mathbf{A}_{s} \mathbf{X}^{(s)} & =\frac{d}{d t} \mathbf{X}^{K-1}+\sum_{s=K-1}^{0} \mathbf{A}_{s} \mathbf{X}^{(s)} \\
& =\frac{d}{d t}\left(\sum_{k=1}^{K} \mathbf{X}_{k}^{(K-1)} \mathbf{C}_{k}\right)+\sum_{s=K-1}^{0} \mathbf{A}_{s}\left(\sum_{k=1}^{K} \mathbf{X}_{k}^{(s)} \mathbf{C}_{k}\right) \\
& =\sum_{k=1}^{K} \mathbf{X}_{k}^{(K)} \mathbf{C}_{k}+\sum_{k=1}^{K} \mathbf{X}_{k}^{(K-1)} \dot{\mathbf{C}}_{k}+\sum_{k=1}^{K}\left(\sum_{s=K-1}^{0} \mathbf{A}_{s} \mathbf{X}_{k}^{(s)} \mathbf{C}_{k}\right) \\
& =\sum_{k=1}^{K}\left(\sum_{s=K}^{0} \mathbf{A}_{s} \mathbf{X}_{k}^{(s)}\right) \mathbf{C}_{k}+\sum_{k=1}^{K} \mathbf{X}_{k}^{(K-1)} \dot{\mathbf{C}}_{k}=\sum_{k=1}^{K} \mathbf{X}_{k}^{(K-1)} \dot{\mathbf{C}}_{k}=0 .
\end{aligned}
$$

Таким образом, на матрицы $C_{k}$ накладьвается $K$ условий, аналогичных тем, что даются в МВП скалярных ЛОДУ:

$$
\sum_{k=1}^{K} \mathbf{X}_{k}^{(q)} \dot{\mathbf{C}}_{k}=0, \quad q=0, \ldots, K-2, \quad \sum_{k=1}^{K} \mathbf{X}_{k}^{(K-1)} \dot{\mathbf{C}}_{k}=\mathbf{F} .
$$


Полученная СМАЛЛУ относительно $\dot{\mathbf{C}}_{k}$ в силу условия (33) является невырожденной. Таким образом, в $M_{K n}$ существует верхний триангулятор [4] T, т.е. диагональная операторная матрица с элементами $\mathbf{T}_{i}^{i}$ :

$$
\mathbf{T}_{1}^{1}=\mathbf{E}, \quad \mathbf{T}_{i}^{i}=\prod_{k=i-1}^{1} \Gamma\left(\mathbf{X}_{k}^{(k-1)}\right), \quad i=2, \ldots, K
$$

который приводит систему (36) к верхнетреугольному виду

$$
\begin{aligned}
& \sum_{k=i}^{K}\left[\prod_{l=1}^{i-1} \Gamma\left(\mathbf{X}_{l}^{(l-1)}\right) \mathbf{X}_{k}^{(i-1)}\right] \dot{\mathbf{C}}_{k}=0, \quad i=2, \ldots, K-1, \\
& \sum_{k=1}^{K} \mathbf{X}_{k} \dot{\mathbf{C}}_{k}=0, \quad \prod_{l=1}^{K-1} \Gamma\left(\mathbf{X}_{l}^{(l-1)}\right) \mathbf{X}_{K}^{(K-1)} \dot{\mathbf{C}}_{K}=\mathbf{F}
\end{aligned}
$$

Если каждое из уравнений этой системы разрешить относительно $\dot{\mathbf{C}}_{k}$ с наименьшим индексом, что в силу условия (33) допустимо, то

$$
\begin{aligned}
&\left(\dot{\mathbf{C}}_{k} \equiv \mathbf{S}_{k}\right) \Longrightarrow \mathbf{S}_{l}=-\left[\prod_{m=1}^{l-1} \Gamma\left(\mathbf{X}_{m}^{(m-1)}\right) \mathbf{X}_{l}^{(l-1)}\right]^{-1} \\
& \times \sum_{i=l+1}^{K}\left[\prod_{m=1}^{l-1} \Gamma\left(\mathbf{X}_{m}^{(m-1)}\right) \mathbf{X}_{i}^{(l-1)}\right] \mathbf{S}_{i}, \quad l=2, \ldots, K-1, \\
& \mathbf{S}_{1}=-\mathbf{X}_{1}^{-1} \sum_{i=2}^{K} \mathbf{X}_{i} \mathbf{S}_{i}, \quad \mathbf{S}_{K}=\left[\prod_{m=1}^{K-1} \Gamma\left(\mathbf{X}_{m}^{m-1}\right) \mathbf{X}_{K}^{(K-1)}\right]^{-1} \mathbf{F} .
\end{aligned}
$$

Это и позволяет записать (32).

Убедиться в общности полученного решения можно, записав $\mathbf{C}_{k}$ в виде

$$
\mathbf{C}_{k}=\mathbf{J}_{k}(t)-\mathbf{J}_{k}\left(t_{0}\right)+\mathbf{Q}_{k}, \quad \mathbf{J}_{k}(t) \equiv \int \mathbf{S}_{k} d t, \quad \dot{\mathbf{Q}}_{k}=0
$$

Тогда $\mathbf{X}=\sum_{k=1}^{K} \mathbf{X}_{k}\left(\mathbf{J}_{k}(t)-\mathbf{J}_{k}\left(t_{0}\right)+\mathbf{Q}_{k}\right)$, а набор начальных условий $\mathbf{X}^{(q)}\left(t_{0}\right)=\mathbf{R}_{q}$ $\left(\dot{\mathbf{R}}_{q}=0\right)$ записьвается как

$$
\sum_{k=1}^{K} \mathbf{X}_{k}^{(q)}\left(t_{0}\right) \mathbf{Q}_{k}=\mathbf{R}_{q}-\left.\frac{d^{q}}{d t^{q}}\left(\sum_{k=1}^{K} \mathbf{X}_{k} \mathbf{J}_{k}(t)\right)\right|_{t=t_{0}}
$$

В силу (33) эта алгебраическая система всегда разрешима. 
ЗАМЕчАниЕ. Подобно скалярному случаю, введением неизвестных $Y_{k} \equiv X^{(k-1)}$ МЛЛОДУ.К может быть сведено к системе матричных левосторонних ЛОДУ.1

$$
\frac{d}{d t}\left(\begin{array}{c}
\mathbf{Y}_{1} \\
\mathbf{Y}_{2} \\
\ldots \\
\mathbf{Y}_{K}
\end{array}\right)=\left(\begin{array}{ccccc}
0 & \mathbf{E} & 0 & \ldots & 0 \\
0 & 0 & \mathbf{E} & \ldots & 0 \\
\ldots \ldots & \ldots & \ldots & \ldots & \ldots \\
-\mathbf{A}_{0} & -\mathbf{A}_{1} & -\mathbf{A}_{2} & \ldots & -\mathbf{A}_{K-1}
\end{array}\right)\left(\begin{array}{c}
\mathbf{Y}_{1} \\
\mathbf{Y}_{2} \\
\ldots \\
\mathbf{Y}_{K}
\end{array}\right) \rightarrow\left(\begin{array}{c}
0 \\
0 \\
\ldots \\
\mathbf{F}
\end{array}\right)
$$

Рассматривая $\mathbf{Y}_{k}=\mathbf{Y}_{k 0}^{l} \mathbf{V}_{l}$, где $\mathbf{Y}_{k 0}^{l}-K$ решений однородной системы $(38)$, имеем $\mathbf{Y}_{k 0}^{l} \dot{\mathbf{V}}_{l}=\mathbf{F}_{k}$, где $\mathbf{F}_{k}=0, k=1, \ldots, K-1, \mathbf{F}_{K}=\mathbf{F} \cdot \operatorname{Taк~как~} \mathbf{Y}_{k 0}^{l} \equiv \mathbf{X}_{l}^{(k-1)}$, последнюю систему можно записать как

$$
\sum_{l=1}^{K} \mathbf{X}_{l}^{(k-1)} \dot{\mathbf{V}}_{l}=\mathbf{F}_{K}
$$

Если произвести замену $(l, k, V) \rightarrow(k, q, C)$, то система принимает вид (36). Следовательно, дополнительньй вариант МВП уравнения (11), следуюший из метода вариации эквивалентной ему СМЛЛОДУ.1, совпадает с тем, что дает теорема 2.

Как и в случае теоремы 1 , поясним действие и символику теоремы 2

СлЕДСТВИЕ. Общими решениями уравнений (22) и (23) являются colon $\mathbf{X}$ :

$$
\begin{aligned}
& \mathbf{X}=-\mathbf{X}_{1} \int \mathbf{X}_{1}^{-1} \mathbf{X}_{2}\left[\Gamma\left(\mathbf{X}_{1}\right) \dot{\mathbf{X}}_{2}\right]^{-1} \mathbf{F} d t+\mathbf{X}_{2} \int\left[\Gamma\left(\mathbf{X}_{1}\right) \dot{\mathbf{X}}_{2}\right]^{-1} \mathbf{F} d t \\
& \ddot{\mathbf{X}}_{k}+\mathbf{A}_{1} \dot{\mathbf{X}}_{k}+\mathbf{A}_{0} \mathbf{X}_{k}=0, \quad\left|\mathbf{X}_{1} \Gamma\left(\mathbf{X}_{1}\right) \dot{\mathbf{X}}_{2}\right| \neq 0, \quad k=1,2,
\end{aligned}
$$

$u$

$$
\begin{aligned}
\mathbf{X}=-\mathbf{X}_{1} \int \mathbf{X}_{1}^{-1}\left\{\mathbf{X}_{2}\left[\Gamma\left(\mathbf{X}_{1}\right) \dot{\mathbf{X}}_{2}\right]^{-1}\left[\Gamma\left(\mathbf{X}_{1}\right) \dot{\mathbf{X}}_{3}\right]-\mathbf{X}_{3}\right\}\left[\Gamma\left(\dot{\mathbf{X}}_{2}\right) \Gamma\left(\mathbf{X}_{1}\right) \ddot{\mathbf{X}}_{3}\right]^{-1} \mathbf{F} d t \\
-\mathbf{X}_{2} \int\left[\Gamma\left(\mathbf{X}_{1}\right) \dot{\mathbf{X}}_{2}\right]^{-1}\left[\Gamma\left(\mathbf{X}_{1}\right) \dot{\mathbf{X}}_{3}\right]\left[\Gamma\left(\dot{\mathbf{X}}_{2}\right) \Gamma\left(\mathbf{X}_{1}\right) \ddot{\mathbf{X}}_{3}\right]^{-1} \mathbf{F} d t \\
+\mathbf{X}_{3} \int\left[\Gamma\left(\dot{\mathbf{X}}_{2}\right) \Gamma\left(\mathbf{X}_{1}\right) \ddot{\mathbf{X}}_{3}\right]^{-1} \mathbf{F} d t \\
\quad \dddot{\mathbf{X}}_{k}+\mathbf{A}_{2} \ddot{\mathbf{X}}_{k}+\mathbf{A}_{1} \dot{\mathbf{X}}_{k}+\mathbf{A}_{0} \mathbf{X}_{k}=0, \quad k=1, \ldots, 3,
\end{aligned}
$$

əде

$$
\begin{aligned}
& \Gamma\left(\mathbf{X}_{1}\right) \dot{\mathbf{X}}_{i} \equiv \dot{\mathbf{X}}_{i}-\dot{\mathbf{X}}_{1} \mathbf{X}_{1}^{-1} \mathbf{X}_{i}, \quad i=1,2,3 \\
& \Gamma\left(\dot{\mathbf{X}}_{2}\right) \Gamma\left(\mathbf{X}_{1}\right) \ddot{\mathbf{X}}_{3} \equiv \Gamma\left(\Gamma\left(\mathbf{X}_{1}\right) \dot{\mathbf{X}}_{2}\right)\left(\Gamma\left(\mathbf{X}_{1}\right) \ddot{\mathbf{X}}_{3}\right) \equiv \ddot{\mathbf{X}}_{3}-\ddot{\mathbf{X}}_{1} \mathbf{X}_{1}^{-1} \mathbf{X}_{3} \\
& \quad-\left(\ddot{\mathbf{X}}_{2}-\ddot{\mathbf{X}}_{1} \mathbf{X}_{1}^{-1} \mathbf{X}_{2}\right)\left(\dot{\mathbf{X}}_{2}-\dot{\mathbf{X}}_{1} \mathbf{X}_{1}^{-1} \mathbf{X}_{2}\right)^{-1}\left(\dot{\mathbf{X}}_{3}-\dot{\mathbf{X}}_{1} \mathbf{X}_{1}^{-1} \mathbf{X}_{3}\right)
\end{aligned}
$$

ДокАЗАТЕЛЬСтво. Если в теореме 2 положить $K=2$, то общее решение системы (1), принимающей вид (22), определяется в виде colon $\mathbf{X}: \mathbf{X}=\mathbf{X}_{1} \mathbf{C}_{1}+\mathbf{X}_{2} \mathbf{C}_{2}$, где $\ddot{\mathbf{X}}_{k}+\mathbf{A}_{1} \dot{\mathbf{X}}_{k}+\mathbf{A}_{0} \mathbf{X}_{k}=0,\left|\mathbf{X}_{1} \Gamma\left(\mathbf{X}_{1}\right) \dot{\mathbf{X}}_{2}\right| \neq 0$, a $\mathbf{C}_{k}$ определяются соотношениями (32). Первое из этих двух равенств вырождается в $\mathbf{C}_{1}=-\int \mathbf{X}_{1}^{-1} \mathbf{X}_{2} \dot{\mathbf{C}}_{2} d t$, а второе принимает вид $\mathbf{C}_{2}=\int\left[\left.\Gamma\left(\mathbf{X}_{1}\right) \dot{\mathbf{X}}_{2}\right|^{-1} \mathbf{F} d t\right.$. Условие (33) означает невырожденность матриц $\mathbf{X}_{1}$ 
и $\Gamma\left(\mathbf{X}_{1}\right) \dot{\mathbf{X}}_{2}$, что обеспечивает нахождение $\mathbf{C}_{k}$ в той части $\mathbf{T}$, где это вьполняется. Дифференцируя $\mathbf{C}_{2}$ и подставляя $\dot{\mathbf{C}}_{2}$ и $\mathbf{C}_{k}$ в $\mathbf{X}$, получаем (39). При этом формула (27) позволяет записать $\Gamma\left(\mathbf{X}_{1}\right) \dot{\mathbf{X}}_{2}=\dot{\mathbf{X}}_{2}-\dot{\mathbf{X}}_{1} \mathbf{X}_{1}^{-1} \mathbf{X}_{2}$.

Решение системы (32) теоремы 2 определяет через решения однородных МЛЛОДУ.3 как colon $\mathbf{X}$ :

$$
\mathbf{X}=\sum_{k=1}^{3} \mathbf{X}_{k} \mathbf{C}_{k}, \quad \sum_{s=3}^{0} \mathbf{A}_{s} \mathbf{X}_{k}^{(s)}=0, \quad\left|\mathbf{X}_{1} \Gamma\left(\mathbf{X}_{1}\right) \dot{\mathbf{X}}_{2} \Gamma\left(\dot{\mathbf{X}}_{2}\right) \Gamma\left(\mathbf{X}_{1}\right) \ddot{\mathbf{X}}_{3}\right| \neq 0 .
$$

При этом формулы (32) расписьваются в виде

$$
\begin{aligned}
& \mathbf{C}_{1}=-\int \mathbf{X}_{1}^{-1}\left(\mathbf{X}_{2} \dot{\mathbf{C}}_{2}+\mathbf{X}_{3} \dot{\mathbf{C}}_{3}\right) d t \\
& \mathbf{C}_{2}=-\int\left[\Gamma\left(\mathbf{X}_{1}\right) \dot{\mathbf{X}}_{2}\right]^{-1}\left[\Gamma\left(\mathbf{X}_{1}\right) \dot{\mathbf{X}}_{3}\right] \dot{\mathbf{C}}_{3} d t \\
& \mathbf{C}_{3}=\int\left[\Gamma\left(\dot{\mathbf{X}}_{2}\right) \Gamma\left(\mathbf{X}_{1}\right) \ddot{\mathbf{X}}_{3}\right]^{-1} \mathbf{F} d t
\end{aligned}
$$

Дифференцируя $\mathbf{C}_{3}$ и подставляя $\dot{\mathbf{C}}_{3}$ в $\mathbf{C}_{2}$, имеем правый сомножитель в формуле (40). Если подставить $\dot{\mathbf{C}}_{2}$ и $\dot{\mathbf{C}}_{3}$ в $\mathbf{C}_{1}$, то тождественные преобразования позволяют записать коэффициент $\mathbf{X}_{1}$ в (40). По определению же (28)

$$
\Gamma\left(\dot{\mathbf{X}}_{2}\right) \Gamma\left(\mathbf{X}_{1}\right) \ddot{\mathbf{X}}_{3}=\Gamma\left(\Gamma\left(\mathbf{X}_{1}\right) \dot{\mathbf{X}}_{2}\right)\left(\Gamma\left(\mathbf{X}_{1}\right) \ddot{\mathbf{X}}_{3}\right) \text {. }
$$

Если расписать согласно $(27) \Gamma\left(\mathbf{X}_{1}\right) \dot{\mathbf{X}}_{2}$ и $\Gamma\left(\mathbf{X}_{1}\right) \ddot{\mathbf{X}}_{3}$ и ввести матричные последовательности $\mathbf{A}_{i}=\Gamma\left(\mathbf{X}_{1}\right) \dot{\mathbf{X}}_{i}$ и $\mathbf{B}_{i}=\Gamma\left(\mathbf{X}_{1}\right) \ddot{\mathbf{X}}_{i}, i=2,3$, то определение произведения операторов Гаусса (28) позволяет представить $\Gamma\left(\dot{\mathbf{X}}_{2}\right) \Gamma\left(\mathbf{X}_{1}\right) \ddot{\mathbf{X}}_{3}$ в указанном виде.

3. Достаточные условия разрешимости в квадратурах систем (1) определяются значительно сложнее, чем в случае отдельных уравнений. Это связано с тем, что к проблемам интегрирования скалярных ЛОДУ.К, обобшаемых системами (1), добавляются специфические проблемы оперирования матричньп уравнением (11). Только при очень жестких ограничениях на параметры МЛЛОДУ.К его решение сводится к решению совокупности скалярных уравнений высших порядков. Примером таких ограничений является, очевидно, диагональность матриц $\mathbf{A}_{s}$. Обобщение этого случая дает

ПРЕДЛОЖЕНИЕ 3. Если в системе (1) над комплексным полем матрицы $\mathbf{A}_{s}$ принадлежат разрешимой алгебре Ли, то ее решение сводится к решению однородных ЛОДУ.К

$$
\sum_{s=K}^{0} a_{i s} y_{i}^{(s)}=0, \quad a_{i s} \in C^{0}(t)
$$

ДокаЗАТЕЛЬСтво. Если все $\mathbf{A}_{s}$ принадлежат разрешимой $L_{N}\left(U_{N-K}=\varnothing\right)$, то согласно теореме Ли [4, с. 62$]$ найдется такая невырожденная матрица T, что преобразование подобия $\mathbf{T} \mathbf{A}_{s} \mathbf{T}^{-1}$ приводит их одновременно к треугольной форме. Так что, умножая (1) слева на $\mathbf{T}$, мы имеем систему

$$
\sum_{s=K}^{0}\left(\mathbf{T A}_{s} \mathbf{T}^{-1}\right)_{i}^{j} y_{j}^{(s)}=T_{i}^{j} f_{j}, \quad y_{j} \equiv T_{j}^{k} x_{k}, \quad \dot{T}_{i}^{j}=0, \quad \mathbf{T} \equiv\left(T_{i}^{j}\right) .
$$


Если $\left(\mathbf{T} \mathbf{A}_{s} \mathbf{T}^{-1}\right)_{i}^{i}=a_{i s}$, то полученное равенство эквивалентно системе

$$
\sum_{s=K}^{0} a_{i s} y_{i}^{(s)}=T_{i}^{j} f_{j},-\sum_{s=K}^{0}\left(\mathbf{T A}_{s} \mathbf{T}^{-1}\right)_{i}^{q} y_{q}
$$

где $q$, в зависимости от вида треугольности, либо меньше, либо больше $i$. Таким образом, МВП позволяет решение системы (1) свести к решению совокупности уравнений (41).

Некоммутативность матричного умножения сильно усложняет процедуру интегрирования МЛЛОДУ.К, сводящуюся часто к ряду нерешенных проблем теории матриц. Тем не менее, некоторые методы классической теории ОДУ могут быть обобщены на матричный случай. Это позволяет дать дополнительные условия разрешимости систем (1). Так теорема о факторизуемости МЛЛОДУ.К [5] позволяет сформулировать

ПРЕДЛОЖЕНИЕ 4. Если в системе (1) $\mathbf{A}_{s}=\mathbf{P}_{K, s}$, a матрицы $\mathbf{P}_{k, s}, k=1, \ldots, K$, индуктивно определяются в виде

$$
\begin{aligned}
\mathbf{P}_{k, k-1} & =\mathbf{B}_{k}+\mathbf{P}_{k-1, k-2}, \quad \mathbf{P}_{k, k}=\mathbf{E}, \\
\mathbf{P}_{k, j} & =\dot{\mathbf{P}}_{k-1, j}+\mathbf{B}_{k} \mathbf{P}_{k-1, j-1}+\mathbf{P}_{k-1, j-1}, \quad j=1, \ldots, K-2, \\
\mathbf{P}_{k, 0} & =\dot{\mathbf{P}}_{k-1, j}+\mathbf{B}_{k} \mathbf{P}_{k-1,0}, \quad \mathbf{P}_{1,0}=\mathbf{B}_{1},
\end{aligned}
$$

әде $\mathbf{B}_{k}=b_{k}^{\alpha} \mathbf{E}_{\alpha}, \alpha=1, \ldots, M, b_{k}^{\alpha} \in \mathbf{C}^{k}(t),\left\|\mathbf{B}_{k}\right\|<\infty$, a $\mathbf{E}_{\alpha}-$ nостоянные матрииы базиса $M$-мерного радикала $R_{M}$ некоторого представления в $M_{n}$ алгебры Ли $L_{N}$, то общим решением системы (1) является colon $\mathbf{X}$ :

$$
\mathbf{X}=\mathbf{Y}_{K} \int \mathbf{Y}_{K}^{-1} \mathbf{Y}_{K-1} \int \cdots \int \mathbf{Y}_{2}^{-1} \mathbf{Y}_{1} \int \mathbf{Y}_{1}^{-1} \mathbf{F} d t \cdots d t
$$

где $\mathbf{Y}_{k}=\exp \left(y_{k}^{\alpha} \mathbf{E}_{\alpha}\right): \mathbf{Y}_{k}(0)=\mathbf{E}$, а $y_{k}^{\alpha}$ находятся в квадратурах из системь

$$
\dot{y}_{k}^{\beta}=-b_{k}^{\gamma}\left[\sum_{n=0}^{\infty} \frac{1}{(n+1) !}\left(y_{k}^{\alpha} C_{\alpha \beta}^{\gamma}\right)^{n}\right]^{-1}, \quad y_{k}^{\alpha}(0)=0 .
$$

ДокАЗАТЕЛьСТво. В работе [6] показано, что матрица $X$ из (43) является решением МЛЛОДУ.К (11) с $A_{s}$ вида (42). Следовательно, решением системы (1) будет любой столбец этой матрищы. Чтобы убедиться в общности его, запишем (43) в виде

$$
\begin{aligned}
\mathbf{X}= & \mathbf{Y}_{K}\left(\int _ { 0 } ^ { t } \mathbf { Y } _ { K } ^ { - 1 } \mathbf { Y } _ { K - 1 } \left(\int _ { 0 } ^ { t _ { 1 } } \cdots \left(\int _ { 0 } ^ { t _ { K - 2 } } \mathbf { Y } _ { 2 } ^ { - 1 } \mathbf { Y } _ { 1 } \left(\int_{0}^{t_{K-1}} \mathbf{Y}_{1}^{-1} \mathbf{F} d t_{K}\right.\right.\right.\right. \\
& \left.\left.\left.\left.+\mathbf{Q}_{1}\right) d t_{K-1}+\mathbf{Q}_{2}\right) \cdots\right) d t_{1}+\mathbf{Q}_{K}\right) .
\end{aligned}
$$

Тогда равенства $\mathbf{X}^{(q)}(0)=\mathbf{R}_{q}: \dot{\mathbf{R}}_{q}=0$ позволяют последовательно явно выражать $Q_{k}$ через $\mathbf{R}_{q}$ и $\mathbf{B}_{k}^{(q)}(0)$. Так $\left(X(0)=R_{0}\right) \Longrightarrow\left(R_{0}=Q_{K}\right)$,

$$
\begin{aligned}
& \left(\dot{X}(0)=R_{1}\right) \Longrightarrow\left(B_{K}(0) Q_{K}+Q_{K-1}=R_{1}\right) \Longrightarrow\left(Q_{K-1}=R_{1}-B_{K}(0) R_{0}\right) \\
& \left(\ddot{\mathbf{X}}(0)=\mathbf{R}_{2}\right) \Longrightarrow\left(\mathbf{R}_{2}=\left(\dot{\mathbf{B}}_{K}(0)+\mathbf{B}_{K}^{2}(0)\right) \mathbf{Q}_{K}+\mathbf{B}_{K-1}(0) \mathbf{Q}_{K-1}+\mathbf{Q}_{K-2}\right) \\
& \quad \Longrightarrow\left(\mathbf{Q}_{K-2}=\mathbf{R}_{2}-\left(\dot{\mathbf{B}}_{K}(0)+\mathbf{B}_{K}^{2}(0)\right) \mathbf{R}_{0}-\mathbf{B}_{K-1}(0)\left(\mathbf{R}_{1}-\mathbf{B}_{K}(0) \mathbf{R}_{0}\right)\right) \ldots
\end{aligned}
$$


Следует отметить, что матрицы $\mathbf{Y}_{k}$ можно находить не только в экспоненциальном, но и в мультипликативно-экспоненциальном виде [6]:

$$
\begin{aligned}
& \mathbf{Y}_{k}=\prod_{\alpha=1}^{M} \exp \left(g_{k \alpha} \mathbf{E}_{\alpha}\right), \quad \mathbf{Y}_{k}(0)=\mathbf{E}, \\
& \dot{g}_{k \alpha}=-b_{k}^{\beta}\left(\prod_{\gamma=1}^{\alpha-1} \exp \left(g_{k \alpha} C_{\gamma \alpha}^{\beta}\right)\right)^{-1}, \quad g_{k \alpha}(0)=0,
\end{aligned}
$$

котороек ряде случаев легче использовать практически.

Случаи $K=2,3$ для МЛЛОДУ.К рассмотрены в [5]. Там же имеется пример.

Более общие, чем в предложении 4, условия разрешимости систем (1) могут быть установлены при рассмотрении МЛЛОДУ.К, эквивалентньх триангулируемым системам матричных уравнений [7]. Для получения таких ограничений необходимо использовать формулы решения левосторонних и правосторонних систем алгебраических линейных уравнений. Первые из них имеют вид (29), а вторые решаются с помощю аналогичных формул, содержащих правосторонние операторы Гаусса [3]. Ввиду громоздкости формулирования и записи подобных ограничений, покажем их вид на примере системы второго порядка.

ПРЕДЛОЖЕНИЕ 5. Если в системе (22) $f_{i}=0$, а матрицы $\mathbf{A}_{s}$ имеют вид

$$
\begin{aligned}
& \mathbf{A}_{1}=-\left(\dot{\mathbf{B}}_{1}^{2}+\mathbf{B}_{1}^{1} \mathbf{B}_{1}^{2}+\mathbf{B}_{1}^{2} \mathbf{B}_{2}^{2}\right)\left(\mathbf{B}_{1}^{2}\right)^{-1}, \\
& \mathbf{A}_{0}=\mathbf{A}_{1} \mathbf{B}_{1}^{1}-\dot{\mathbf{B}}_{1}^{1}-\left(\mathbf{B}_{1}^{1}\right)^{2}-\mathbf{B}_{1}^{2} \mathbf{B}_{2}^{1},
\end{aligned}
$$

əде $\mathbf{B}_{k}^{p} \in M_{n}^{1}(t), k, l=1,2,\left|\mathbf{B}_{1}^{2}\right| \neq 0$

$$
\begin{aligned}
& \mathbf{B}_{1}^{1}=\left[\left(\mathbf{T}_{1}^{1} \mathbf{C}_{1}^{1}+\mathbf{T}_{1}^{2} \mathbf{C}_{2}^{1}\right)\left(\mathbf{E}+\left(\mathbf{T}_{1}^{1}\right)^{-1} \mathbf{T}_{1}^{2} \mathbf{H}^{-1} \mathbf{T}_{1}^{1}\right)-\mathbf{T}_{1}^{2} \mathbf{C}_{2}^{2} \mathbf{H}^{-1} \mathbf{T}_{2}^{1}\right]\left(\mathbf{T}_{1}^{1}\right)^{-1}, \\
& \mathbf{B}_{1}^{2}=-\left(\mathbf{T}_{1}^{1} \mathbf{C}_{1}^{1}+\mathbf{T}_{1}^{2} \mathbf{C}_{2}^{1}\right)\left(\mathbf{T}_{1}^{1}\right)^{-1} \mathbf{T}_{1}^{2} \mathbf{C}_{2}^{2} \mathbf{H}^{-1}, \\
& \mathbf{B}_{2}^{1}=\left(\mathbf{T}_{2}^{1} \mathbf{C}_{1}^{1}+\mathbf{T}_{2}^{2} \mathbf{C}_{2}^{1}\right)\left[\mathbf{E}+\left(\mathbf{T}_{1}^{1}\right)^{-1} \mathbf{T}_{1}^{2} \mathbf{H}^{-1} \mathbf{T}_{2}^{1}\right]\left(\mathbf{T}_{1}^{1}\right)^{-1}-\mathbf{T}_{2}^{2} \mathbf{C}_{2}^{2} \mathbf{H}^{-1} \mathbf{T}_{2}^{1}, \\
& \mathbf{B}_{2}^{2}=-\left(\mathbf{T}_{2}^{1} \mathbf{C}_{1}^{1}+\mathbf{T}_{2}^{2} \mathbf{C}_{2}^{1}\right)\left(\mathbf{T}_{1}^{1}\right)^{-1} \mathbf{T}_{1}^{2} \mathbf{H}^{-1}+\mathbf{T}_{2}^{2} \mathbf{C}_{2}^{2} \mathbf{H}^{-1},
\end{aligned}
$$

причем $\mathbf{H}=\Gamma\left(\mathbf{T}_{1}^{1}\right) \mathbf{T}_{2}^{2},|\mathbf{H}| \neq 0, \mathbf{T}_{i}^{j}=0, \mathbf{C}_{k}^{k}=\mathbf{C}_{k}^{\alpha} \mathbf{E}_{\alpha}$, a $\mathbf{E}_{\alpha}-$ базис радикала $R_{k}$ алгебры Ли $L_{N}$ над $M_{n}$, то решением этой системы является colon $\left(\mathbf{T}_{1}^{j} \mathbf{Y}_{j}\right): \mathbf{Y}_{1} \equiv$ $\mathbf{Y}_{10} \mathbf{K}_{1}, \mathbf{Y}_{2}=\mathbf{Y}_{20} \int \mathbf{Y}_{20}^{-1} \mathbf{C}_{2}^{1} \mathbf{Y}_{1} d t, \dot{\mathbf{K}}_{1}=0, \mathbf{Y}_{k 0}=\exp \left(y_{k}^{\alpha} \mathbf{E}_{\alpha}\right)$, әде y y $_{k}^{\alpha}$ нахолтся $\boldsymbol{\theta}$ квадратурах из системы (44), в которой произведена замена $\left(b^{\alpha}\right) \rightarrow\left(-c_{k}^{\alpha}\right)$.

ДокАЗАТЕЛЬСТво. Рассмотрим систему однородных МЛЛОДУ.1

$$
\dot{\mathbf{X}}_{k}=\mathbf{B}_{k}^{l} \mathbf{X}_{l}, \quad \mathbf{B}_{k}^{l} \in M_{n}^{1}(t), \quad k, l=1,2, \quad\left|\mathbf{B}_{k}^{\bar{k}}\right| \neq 0, \quad k \neq \bar{k}=1,2 .
$$

Эта система Эквивалентна двум матричным уравнениям второго порядка

$$
\begin{gathered}
\ddot{\mathbf{X}}_{k}+\mathbf{A}_{1 k} \dot{\mathbf{X}}_{k}-\left(\mathbf{A}_{1 k} \mathbf{B}_{k}^{k}+\dot{\mathbf{B}}_{k}^{k}+\mathbf{B}_{k}^{l} \mathbf{B}_{l}^{k}\right) \mathbf{X}_{k}=0 \\
\mathbf{A}_{1 k}=-\left(\dot{\mathbf{B}}_{k}^{\bar{k}}+\mathbf{B}_{k}^{l} \mathbf{B}_{l}^{\bar{k}}\right)\left(\mathbf{B}_{k}^{\bar{k}}\right)^{-1}
\end{gathered}
$$


При $k=1$ коэффициенты МЛЛОДУ.2 принимают вид (45). Так что в силу предложения 2 , теорем 1 и 2 , интегрируемость системы (1) с указанными ограничениями на $\mathbf{A}_{s}$, обеспечивается разрешимостью системы (47). Однако, если в последней сделать замену $\mathbf{X}_{k}=\mathbf{T}_{k}^{l} \mathbf{Y}_{l}$, где $d(\mathbf{T}) \neq 0, \dot{\mathbf{T}} \equiv\left(\dot{\mathbf{T}}_{k}^{l}\right)=0, \mathbf{Y}_{l}$ удовлетворяют матричной системе $\dot{\mathbf{Y}}_{l}=\mathbf{C}_{l}^{k} \mathbf{Y}_{k}$, в которой $\mathbf{C}_{1}^{2}=0$, а $\mathbf{C}_{k}^{k}$ принадлежат $R_{k}$, то $\mathbf{Y}_{k}$ могут быть найдены в квадратурах. Второе уравнение этой системы, $\dot{\mathbf{Y}}_{2}=\mathbf{C}_{2}^{1} \mathbf{Y}_{2}+\mathbf{C}_{2}^{2} \mathbf{Y}_{2}$, имеет решение $\mathbf{Y}_{2}=\mathbf{Y}_{20} \int \mathbf{Y}_{20}^{-1} \mathbf{C}_{2}^{1} \mathbf{Y}_{1} d t$, в котором $\dot{\mathbf{Y}}_{20}=\mathbf{C}_{2}^{2} \mathbf{Y}_{20}$. Если определить $\mathbf{Y}_{k 0}$ в экспоненциальных видах $\mathbf{Y}_{k 0}=\exp \left(y_{k}^{\alpha} \mathbf{E}_{\alpha}\right)$, то на $y_{k}^{\alpha}$ наложатся требования нелинейной системы (44), где функции $b^{\alpha}$ должны быть заменены на $-c_{k}^{\alpha}[6]$. Для $R_{K}$ эта система разрешима в квадратурах. Таким образом, интегрируемость системы (47) обеспечивается требованием $\mathbf{B}_{k}^{l} \mathbf{T}_{l}^{m}=\mathbf{T}_{k}^{l} \mathbf{C}_{l}^{m}$, вытекающим из (47) после подстановки вместо $\mathbf{X}_{k}$ матриц $\mathbf{T}_{k}^{l} \mathbf{Y}_{l}$. А так как в (46) записано решение с помощью формул Фробениуса [8, c. 59] последней алгебраической системы, то условия (46) обеспечивают триангуляцию и интегрируемость системы (47).

Следовательно, colon $\mathbf{X}_{1}$ является решением однородной системы (22).

Следует, видимо, отметить, что определение $\mathbf{B}_{k}^{l}$ с точностью до нумерации позволяет в утверждении предложения 6 рассматривать оба вида МЛЛОДУ.2 (48) и оба вида треугольности матрицы $\mathbf{C}=\left(\mathbf{C}_{k}^{l}\right)$.

Примером, иллюстрирующим предложение 5 , может служить расписанный по компонентам пример работы [7].

Обобщение этого результата на случай систем более высокого порядка формулируется в символах левосторонних и правосторонних операторов Гаусса [3].

\section{СПИСОК ЦИТИРОВАННОЙ ЛИТЕРАТУРЫ}

[1] Понтрягин Л. С. Обыкновенные дифференциальные уравнения. М.: Наука, 1970.

[2] Риордан Д. Комбинаторные тождества. М.: Наука, 1982.

[3] Деревенский В.П. Решение систем матричных алгебраических линейных уравнений // Изв. вузов. Матем. 1998. № 10 (437). С. 32-36.

[4] Джекобсон Н. Алгебры Ли. М.: Мир, 1964.

[5] Деревенский В. П. Матричные линейные дифференциальные уравнения высших порядков // Дифференц. уравнения. 1993. Т. 29. №4. С. 711-714.

[6] Деревенский В. П. Экспоненциальное решение матричных линейных дифференциальных уравнений первого порядка // Изв. вузов. Матем. 1981. № 7. С. 31-33.

[7] Деревенский В. П. Системы матричных линейных дифференциальных уравнений первого порядка // Матем. заметки. 1999. Т. 66. №1. С. 63-75.

[8] Гантмахер Ф.Р. Теория матриц. М.: Наука, 1967. 